\title{
A Propensity Score Matched Approach to Assess the Associations of Commonly Prescribed Medications with Fall Risk in a Large Harmonized Cohort of Older Ambulatory Persons
}

\author{
L. J. Seppala' ${ }^{1}$ B. van de Loo ${ }^{1,3} \cdot$ M. Schut ${ }^{2}$ - N. M. van Schoor ${ }^{3}$ B. H. Stricker ${ }^{4}$ R. A. Kenny ${ }^{5}$ F. Moriarty ${ }^{5,6}$. \\ L. C. P. G. M. de Groot ${ }^{7} \cdot$ M. Denkinger ${ }^{8} \cdot$ D. Rothenbacher ${ }^{9} \cdot$ Nathalie van der Velde $^{1}$ (1) $\cdot$ A. Abu-Hanna ${ }^{2}$
}

Accepted: 5 June 2021 / Published online: 5 July 2021

(c) The Author(s) 2021

\begin{abstract}
Introduction Several medication classes are considered to present risk factors for falls. However, the evidence is mainly based on observational studies that often lack adequate adjustment for confounders. Therefore, we aimed to assess the associations of medication classes with fall risk by carefully selecting confounders and by applying propensity score matching (PSM). Methods Data from several European cohorts, harmonized into the ADFICE_IT cohort, was used. Our primary outcome was time until the first fall within 1-year follow-up. The secondary outcome was a fall in the past year. Our exposure variables were commonly prescribed medications. We used 1:1 PSM to match the participants with reported intake of specific medication classes with participants without. We constructed Cox regression models stratified by the pairs matched on the propensity score for our primary outcome and conditional logistic regression models for our secondary outcome.

Results In total, $32.6 \%$ of participants fell in the 1-year follow-up and $24.4 \%$ reported falling in the past year. ACE inhibitor users (prevalence of use 15.3\%) had a lower fall risk during follow-up when matched to non-users, with a hazard ratio (HR) of 0.82 (95\% CI 0.68-0.98). Also, statin users (prevalence of use 20.1\%) had a lower risk, with an HR of 0.76 (95\% CI 0.65-0.90). Other medication classes showed no association with risk of first fall. Also, in our secondary outcome analyses, statin users had a significantly lower risk. Furthermore, $\beta$-blocker users had a lower fall risk and proton pump inhibitor use was associated with a higher risk in our secondary outcome analysis.

Conclusion Many commonly prescribed medication classes showed no associations with fall risk in a relatively healthy population of community-dwelling older persons. However, the treatment effects and risks can be heterogeneous between individuals. Therefore, focusing on identification of individuals at risk is warranted to optimize personalized falls prevention.
\end{abstract}

\section{Introduction}

Falls are one of the most common health problems facing older persons today. Approximately one third of community dwellers over the age of 65 years will fall at least once yearly and almost $50 \%$ of them will experience a recurrent fall within the next year [1]. In one out of five fall incidents, serious injury such as fracture or head trauma occurs [2]. Also, even falls without an injury can have significant negative effects on the quality of life of an older person, since falls have been associated with greater functional decline, social withdrawal, fear of falling and symptoms of anxiety

Nathalie van der Velde

n.vandervelde@amsterdamumc.nl

Extended author information available on the last page of the article

\section{Key Points}

Use of certain medications and polypharmacy are considered risk factors for falls. In general, the evidence is based on data of observational studies with several quality issues.

We found no associations between many commonly prescribed medication classes and fall risk in a relatively healthy population of older adults.

In terms of clinical practice, deprescribing of appropriate medication in healthy older persons without side effects is likely not judicious in falls prevention.

The results of this study cannot be generalized to frail older adults and there is a need for studies assessing the medication-related fall risk in frail populations. 
and depression [1,3]. Fear of falling has an adverse impact on functioning, quality of life, mental health and social network and has been associated with mortality and cognitive impairment among others $[4,5]$. Besides the impact on an individual, fall incidents and related injuries cause substantial burden to societies and health care systems. Fall-related costs have been estimated to vary between $0.85 \%$ and $1.5 \%$ of total health care expenditures of Western countries [6].

In older adults, falls tend to result not from a single but from several interacting risk factors [7]. One of the important and modifiable risk factors is the use of certain medications, so-called fall-risk-increasing drugs (FRIDs) [8-14]. To date, several systematic reviews and meta-analyses have summarized the evidence over the past decades. In particular, psychotropics are viewed as important FRIDs [9]. In general, the evidence is based on data from observational studies, since falls have not been standardly assessed as adverse events in drug trials (randomized controlled trials, RCTs) [15]. These observational studies often have several quality issues such as small sample size, a lack of a precise definition of falls or the studied target medication and lack of careful and adequate adjustment for confounders [15]. Therefore, well designed observational studies are still warranted [15].

Moreover, current falls prevention guidelines emphasize that older adults at high risk of falling should be assessed for FRIDs and that medication review including modification or withdrawal of FRIDs should be considered [16]. Since the established and potential FRIDs are commonly prescribed medications in older persons with potentially beneficial effects with regard to symptom and disease control, it is essential to know with more certainty which drug classes are causally related to fall risk. However, conducting an $\mathrm{RCT}$, the gold standard of causality, may be unethical and even if data on falls is collected, RCTs might be underpowered to detect the existing relationships. Second, often the participants selected in RCTs do not represent real-world patients due to stringent inclusion criteria. Observational studies are hence an attractive source for evidence, but the evidence coming from observational studies has to be carefully evaluated to assure that study design and analysis features have been carefully chosen to minimize bias and confounding [15]. Therefore, proper adjustment for confounding factors would give more accurate insights into the relationships between medications and fall risk. Propensity scoring is a statistical approach for observational data aimed at creating and comparing groups with similar treatment or exposure probability [17]. The propensity scoring in this context is defined as a subject's probability of receiving a specific treatment conditional on the observed covariates. One way to use it is by building strata of matched treated and non-treated patients based on their propensity score. It helps in choosing similar patient groups for comparison and, importantly, a matched data set allows transparent analyses. Therefore, our aim was to investigate the associations of commonly prescribed medication classes and fall risk by applying propensity score matching in a large, harmonized cohort of relatively healthy older adults.

\section{Methods}

\subsection{Study Population}

For the current study, we used data from five European cohort studies, harmonized into a single large cohort in the ADFICE_IT project [18]. The studies and cohorts included are reviewed briefly below.

The Rotterdam study is an ongoing prospective followup study in three cohorts since 1990, and as of 2008, the study comprised 14,926 participants of 50 years and older originating from the Ommoord area located in Rotterdam [19]. The harmonized cohort contains data from the fifth examination cycle of the first cohort, the third examination cycle of the second cohort and the second examination cycle of the third cohort. These examination cycles took place between 2009 and 2013.

The B-PROOF is a large, multicentre, randomized, double-blind, placebo-controlled trial $(n=2919)$ conducted in the Netherlands in which persons of 65 years and older with elevated homocysteine levels were included from 2008 to 2011 [20]. The primary aim was to assess whether vitamin B12 and folic acid is effective in the prevention of osteoporotic fractures. No difference was found between the groups for any of the fall outcomes [21]. Therefore, it is reasonable to harmonize B-PROOF with the other cohorts.

The Longitudinal Aging Study Amsterdam (LASA) is a prospective cohort study that started in 1992 and included older persons aged between 55 and 85 years [22]. A random sample was drawn from the population of 11 municipalities of the Netherlands and was stratified by age and sex. Initiated by the Dutch government, it aims to identify predictors and consequences of ageing. The harmonized cohort contains data from the second examination cycle of the first cohort $(n=2545)$, for which the observation was between 1995 and 1996 and from which 1509 respondents participated in the fall follow-up study. Furthermore, it contains data from the baseline observation of the third cohort $(n=1023)$, which was performed between 2012 and 2013.

The study on Activity and Function in the Elderly in Ulm (ActiFE-Ulm) is a population-based cohort study consisting of a random sample of 7460 persons aged 65 years and over who were selected from the population registers in Ulm, Neu-Ulm and an adjacent region 
(Alb-Donau-Kreis) in Germany [23]. The recruitment phase started in February 2009 and finished in April 2010. In total, 1506 persons agreed to participate in the study. The primary focus was physical activity, measured by sensor technology, and the consequences of physical activity for cognitive, emotional and social functioning.

The Irish Longitudinal Study on Ageing (TILDA) is an ongoing cohort study recruited via random sampling to receive a representative sample of aging adults in Ireland [24]. Baseline data from the 8504 primary and secondary participants aged 50 years and over were collected between October 2009 and July 2011 and these baseline data were included in the harmonized cohort.

All participants provided written informed consent, and all studies were approved by their respective institutional ethics committees [19, 20, 22-24].

\subsection{Outcome}

The primary outcome for the current study in the harmonized dataset was time to first fall in 12 months' follow-up measured in weeks. A fall was defined as an unintentional change in position resulting in coming to rest at a lower level or on the ground. This data was available for ActiFEUlm, B-PROOF and the first LASA cohort [23, 25]. Participants received fall calendars and were asked to record fall events weekly. Subsequently, they were asked to mail the calendar to the respective study centre at the end of every 3 months. They were contacted by phone if no calendar was returned or if the data was incomplete.

Fall occurrence in the last 12 months at study visit was used as a secondary outcome measure. This data was available for all cohorts. The occurrence of falls in the past 12 months was based on retrospective self-reporting, and a dichotomous outcome variable 'yes/no' was created.

\subsection{Exposure}

In the harmonized dataset, Anatomical Therapeutic Classification (ATC) codes were used to classify drug exposure variables at baseline recruitment [26]. Variables of interest were potential FRIDs [8-10] that were used by at least $5 \%$ of the participants. This included benzodiazepine and related drugs, ACE inhibitors, $\alpha$-blocker used for prostate hyperplasia, calcium channel blockers, angiotensin II receptor blockers, high ceiling diuretics, low ceiling diuretics, $\beta$-blockers, nonsteroidal anti-inflammatory drugs, proton pump inhibitors and statins. The corresponding ATC codes for each medication class are provided in Appendix I (Supplementary Table 1, see electronic supplementary material [ESM]).
The ascertainment of medication use was done during the respective study visits in each cohort. In the Rotterdam study, medication use was registered during the home interview by participants presenting all the medication they were using at the day of the interview [27]. In B-PROOF, a questionnaire was used to ascertain the selfreported medication use [28]. In LASA, interviewers asked participants to show containers of prescription medication they had used in the 2 weeks prior to the interview [29]. In TILDA, medication data were assessed during the home interview with participants showing or reporting all medications taken on a regular basis [30]. In ActiFE-Ulm, barcodes of each pharmaceutical available in participants' homes and taken at baseline visits by the participants were scanned. Products without an available code were entered by hand [23].

\subsection{Covariables}

Sociodemographic variables included sex, age and living status and lifestyle variables included alcohol use and body mass index. The majority of the self-reported chronic diseases were assessed using questionnaires. Also, depressive symptoms, blood pressure and cognitive status were measured. Co-medication use was determined in each cohort as described above. A detailed description of the covariable assessment and the harmonization procedure is presented in Appendix I under subheading 'Covariate Assessment' and in Supplementary Table 2 of the Appendix I (see ESM). The core principle of the harmonization was to capture the most common denominator.

\subsection{Statistical Methods}

We used propensity score matching and in particular, assessed the average treatment effect on the treated (ATT) [31]. In general, the propensity score is the probability of treatment assignment given a set of observed baseline characteristics. Thus, for example in this study, each participant has a certain probability of using statins, thus a propensity score for statin use. This score is obtained by a logistic regression model. The model predicts the probability of receiving treatment, thus the propensity score, based on the set of an individual's observed baseline characteristics. The propensity score is the basis for the matching procedure. Each treated person is matched to one (or more) non-treated persons based on their propensity scores (details follow). Finally, the effect of treatment on the outcome is estimated in the propensity score matched sub-sample.

First, before propensity score matching, we assessed baseline characteristics in the treated and the untreated groups by calculating frequencies and means. To account for missing covariables, missing data, which we considered as missing at 
random, was imputed in five copies with multivariate imputations by chained equations [32]. The median of the missing values for the covariables excluding variables without missing variables was 4993 in the cross-sectional dataset (interquartile range [IQR] 2977-9678) and 686 (IQR 163-2918) in the prospective dataset. Also, standardized mean differences (SMD) were calculated between the groups for each variable in each imputed copy of the dataset. For each variable, we reported its highest SMD (most imbalanced) and the percentages or means from the dataset with the highest SMD of all five imputed copies.

Second, propensity scores (PS) were estimated by conducting logistic regression models separately for each exposure. The different medication classes were the response variables for these models. The selection of covariates for the PS model, fall-risk factors and confounders, was based on clinical knowledge and literature a priori $[31,33]$. In addition, cohort index and possible alternative therapies were included in the models. For example, in case of calcium channel blocker analysis, other antihypertensives were considered alternative therapies. We excluded possible intermediates between medication classes and fall risk from the models, thus the variables included in the models differed for each exposure. PS models were conducted in each imputed copy of the dataset, and means of the propensity scores were calculated.

Third, for the PS matching, a greedy matching algorithm was used, and the matching was performed on the logit (i.e. the natural logarithm of the odds) of the mean PS of the imputed copies of the dataset. We conducted 1:1 matching without replacement and without callipers.

Fourth, after matching, the balance between the matched, treated and untreated groups was analysed by investigating the SMD for each variable in each copy of the dataset. Just like before matching, we also reported the highest SMD for each variable of all imputed copies after the matching. SMD $<0.1$ was considered an adequate balance for a variable between the treated and untreated groups. In addition, values of SMD $>0.2$ are considered as a serious imbalance.

Finally, as outcome models, we constructed Cox regression models stratified (i.e. clustered) by the pairs matched on the propensity score for time to first fall during 1-year follow-up to assess the association between each exposure variable and fall risk. Conditional logistic regression models were conducted for our secondary outcome, fall risk in the past 12 months. To adjust for possible unbalanced variables (with the SMD $>0.1$ for a certain variable at least in three copies of the datasets) after matching, we obtained doubly robust estimates by including such variables as additional covariates in the outcome models. The associations from the imputed copies of the datasets were pooled using Rubin's rules. All the analyses were performed with the statistical environment R [34] using packages mice, tableone, Matching and survival.

\section{Results}

\subsection{Prospective Data}

Baseline characteristics and SMDs of the matched, treated and untreated groups before and after matching are shown in Supplementary Tables 3-13 in Appendix 2 of the ESM separately for each exposure medication class. The cohort contained prospective data for 5722 participants before matching. In general, the balance was adequate after matching. Most of the analyses had some covariates with SMD between 0.1 and 0.2. Only the diabetes variable was seriously imbalanced (SMD >0.2) after matching statin users to non-users. The associations of medication classes on fall risk using prospective data are found in Table 1. ACE inhibitor users had a lower fall risk when matched to non-users, with a hazard ratio (HR) of 0.82 (95\% CI 0.68-0.98). Also, statin users had a lower risk, with an HR of 0.76 (95\% CI $0.65-0.90)$. Other medication classes showed no statistically significant associations.

\subsection{Cross-Sectional Data}

Baseline characteristics and SMDs of the matched, treated-untreated groups before and after matching are shown in Supplementary Tables 14-23 in Appendix 2 of the ESM separately for each exposure medication class. The cohort contained cross-sectional data for 21,282 participants before matching. In general, in most of the analyses, balance was adequate after matching. However, diabetes and number of used medication variables were seriously imbalanced after matching statin users to non-users. In addition, the arrhythmia variable was seriously imbalanced after matching $\beta$-blocker users to non-users. The associations of the different medication classes with fall risk in the past 12 months are found in Table 2. Statin users had a lower risk, with an OR of 0.84 (95\% CI 0.77-0.93). Furthermore, $\beta$-blocker users had a lower fall risk, with an OR of 0.86 (95\% CI 0.79-0.95). Finally, proton pump inhibitor use was associated with a higher risk, with an OR of 1.16 (95\% CI $1.04-1.30)$. Other medication classes showed no statistically significant associations.

\section{Discussion}

In this propensity score matched approach using data from a harmonized cohort of six European cohorts, we assessed the associations of commonly prescribed medication classes 
with fall risk. In our primary analysis, using prospective data, ACE inhibitor and statin users had a lower fall risk in a follow-up when matched to non-users. Other medication classes showed no significant associations. Also, in our secondary outcome analysis using cross-sectional data, statin users had a lower fall risk in line with our prospective results. Furthermore, $\beta$-blocker users had a lower fall risk. Finally, proton pump inhibitor use was associated with a higher fall risk.

To our knowledge, this is one of the first studies that has used propensity score matching when investigating the associations of medication classes with fall risk. Most of the effect sizes found in this study are comparable to the pooled estimates of recent meta-analyses on this topic [8-10]. Statin use was associated with lower fall risk in both of our analyses (HR 0.76 [0.65-0.90] and OR 0.84 [0.77-0.93]). This is in line with the previous findings from literature in which statin use has been reported to be associated with lower risk in a meta-analysis of unadjusted data (OR 0.80 [0.65-0.98], 3 studies) [8]. Also, ACE inhibitor users had a lower fall risk during followup (HR 0.82 [0.68-0.98]), while in the meta-analysis of

Table 1 Effect of medication use on the falls risk-prospective data

\begin{tabular}{lll}
\hline Medication class & $N$ & Hazard ratio $(95 \% \mathrm{Cl})$ \\
\hline ACE inhibitors & 877 & $0.82(0.68-0.98)^{\mathrm{a}}$ \\
Calcium channel blockers & 699 & $0.94(0.76-1.16)$ \\
Angiotensin II receptor blockers & 629 & $1.06(0.85-1.33)^{\mathrm{b}}$ \\
Low ceiling diuretics & 891 & $0.99(0.82-1.18)^{\mathrm{c}}$ \\
High ceiling diuretics & 394 & $0.88(0.66-1.16)^{\mathrm{d}}$ \\
$\beta$-Blockers & 1406 & $0.98(0.84-1.13)^{\mathrm{e}}$ \\
Statins & 1148 & $0.76(0.65-0.90)^{\mathrm{f}}$ \\
$\alpha$-Blockers for prostate hyperplasia & 295 & $0.85(0.61-1.18)^{\mathrm{g}}$ \\
Nonsteroidal anti-inflammatory drugs & 449 & $1.17(0.92-1.49)$ \\
Proton pump inhibitors & 832 & $1.12(0.93-1.34)$ \\
Benzodiazepines and related drugs & 420 & $1.14(0.89-1.47)^{\mathrm{h}}$ \\
\hline
\end{tabular}

$N$ number of users matched 1:1 to controls

${ }^{a}$ Unbalanced variable diuretics use included in the outcome model

${ }^{\mathrm{b}}$ Unbalanced variables diuretics use, cohort index and hearing problem included in the outcome model

${ }^{\mathrm{c}}$ Unbalanced variable angiotensin II receptor blocker use included in the outcome model

${ }^{\mathrm{d}}$ Unbalanced variables age, living situation, fear of falling, arrhythmia, mini-mental sate examination (MMSE) and hearing problem included in the outcome model

${ }^{\mathrm{e}}$ Unbalanced variables number of medications and use of cardiac vasodilators included in the outcome model

${ }^{\mathrm{f}}$ Unbalanced variables diabetes and number of used medications included in the outcome model

${ }^{\mathrm{g}}$ Unbalanced variables alcohol use, cohort index and anticholinergics use included in the outcome model

${ }^{\mathrm{h}}$ Unbalanced variable fear of falling included in the outcome model
Table 2 Effect of medication use on the falls risk-cross-sectional data

\begin{tabular}{lll}
\hline Medication class & $N$ & Odds ratio $(95 \% \mathrm{Cl})$ \\
\hline ACE inhibitors & 3038 & $0.92(0.82-1.03)$ \\
Calcium channel blockers & 2186 & $0.95(0.83-1.08)$ \\
Angiotensin II receptor blockers & 2544 & $0.97(0.85-1.10)$ \\
Low ceiling diuretics & 2899 & $0.97(0.86-1.09)$ \\
High ceiling diuretics & 1114 & $1.00(0.84-1.20)^{\mathrm{a}}$ \\
$\beta$-Blockers & 4343 & $0.86(0.79-0.95)^{\mathrm{b}}$ \\
Statins & 5555 & $0.84(0.77-0.93)^{\mathrm{c}}$ \\
$\alpha$-Blocker used for prostate hyperplasia & 707 & $0.82(0.65-1.04)$ \\
Proton pump inhibitors & 3601 & $1.16(1.05-1.28)$ \\
Benzodiazepine and related drugs & 1579 & $1.09(0.93-1.26)$ \\
\hline
\end{tabular}

$N$ number of users matched 1:1 to controls

${ }^{\mathrm{a}}$ Unbalanced variable mini-mental sate examination (MMSE) included in the outcome model

${ }^{\mathrm{b}}$ Unbalanced variables arrhythmia, heart failure, and cardiac vasodilators use included in the outcome model

${ }^{\mathrm{c}}$ Unbalanced variables diabetes, number of medication and cardiac vasodilators use included in the outcome model

adjusted data, no significant association was found (OR 0.91 [0.78-1.08], 4 studies) [8]. Furthermore, $\beta$-blocker users had a lower fall risk in our cross-sectional analysis (OR 0.86 [0.79-0.95]) in line with the meta-analysis of adjusted data (OR 0.88 [0.80-0.97], 8 studies) [8]. Finally, proton pump inhibitor use was associated with a higher risk only in a cross-sectional analysis and it should be noted that the vast majority of the European expert panel did not considered proton pump inhibitors to increase fall risk in a consensus effort [35]. This indicates that the found association might be explained by the residual confounding. Proton pump inhibitor use could, for example, be a marker for (multi)morbidity. On the other hand, there is some evidence that it may increase fracture risk, which overlaps with the variable fall risk [36].

Only the effect estimates for high-ceiling diuretics and benzodiazepines and related drugs were markedly lower than in the recent meta-analyses $[8,9]$. These differences could be explained by several factors. First, numerous variables were included in our propensity score and the variable selection of the multivariate models of the previous studies is very heterogeneous [8-10]. Thus, the comparison of the different models is difficult. In theory, by applying propensity score matching and careful confounder selection we may have taken possible residual confounding of earlier studies better into account. Furthermore, in general, propensity score methods tend to give effect sizes modestly nearer to the null compared with multivariate models when both methods have been used to investigate associations in a specific cohort [37]. However, even when applying a Cox 
regression adjusted only for number of used medications, age and gender, we observed markedly lower effect estimates than in the recent meta-analyses (data not shown) [8,9]. Considering benzodiazepines and related drugs, the effect size was heterogeneous between the three cohorts with prospective data. In ActiFE-Ulm, the prevalence of use for these medications and the effect estimate were markedly lower than in the two other cohorts. In general, substantial heterogeneity between the results of different cohorts is often present $[8,9]$. The underlying differences and the explaining factors of heterogeneity between the studies can be related to frailty status of the population, used medication dosage and the indication for the drugs. Moreover, the heterogeneity can reflect differences in the prevalence of use of specific drugs, as broader medication classes are being evaluated in the studies. Also, in our study, there are factors leading to clinical heterogeneity between the cohorts such as age and presence of co-morbidities but also most likely differences in prescription patterns between different countries and between time periods.

Furthermore, many of the commonly prescribed medication classes, such as different antihypertensives, showed no significant associations with fall risk in our study. This finding, in line with previous research, indicates that populationattributable fall risk related to these medication classes on average is low in a population of relatively healthy older persons. The lack of associations with fall risk may well be driven by the positive effects of these medications with regard to symptom management and disease control in this relatively healthy population, as the related clinical conditions can also lead to falls [38]. Although, it should be noted that use of antihypertensives can lead to hypotension and orthostatic hypotension, which both have been linked to increased fall risk [38, 39]. However, risk of adverse drug reactions (ADRs) is strongly dependent on patient characteristics such as multimorbidity, polypharmacy, age and frailty [40]. Thus, given our findings, in terms of clinical practice, deprescribing of appropriate medications in healthy older persons without side effects is likely not judicious in falls prevention. But this conclusion cannot be drawn for frailer populations, since our findings cannot be generalized to frailer older populations like, for example, those visiting falls clinics. Hence, there is an urgent need for studies assessing the fall risk related to these medications in frail populations. In addition, modern data-driven methods can be of aid to estimate the heterogeneous treatment effects and identify the subgroups for whom the treatment generates adverse effects.

Finally, although we carefully examined the inclusion of confounders in our propensity score models, it should be noted that use of a propensity score will not correct biases from unmeasured confounding. In comparison with randomization, the propensity score does not share the ability to balance all confounders, including unmeasured confounders. Most likely, some underlying differences between the treated and untreated groups are still present after propensity score matching. These differences can be related to, for example, treatment decisions of physicians, like the indication or disease severity. For instance, in the case of benzodiazepines, we could not match for sleeping disorders or severity of sleeping disorders. Furthermore, the participants that have suffered from side effects such as falls may have stopped the use of that specific medication. These high-risk participants that have stopped may end up being the non-treated control group in the propensity score analysis, causing survival bias. For example, statin users with muscle-related side effects will stop the use of statins and the users in the treated group are chronic users without side effects. Therefore, targeting new users of specific medications as a study population would be of interest. Finally, we did not account for the effect of dosages in the analyses and it is possible that low and high dosages of certain drugs have different effects in terms of fall risk.

\subsection{Strengths and Limitations}

There are several strengths and limitations related to this study. First, the gold standard of falls measurement, a falls calendar, was used to collect falls data in the cohorts with prospective data. Retrospective self-reporting of falls, such as our secondary outcome measure, leads to under-reporting of falls. However, we believe it is appropriate to report the results of the secondary outcome as this outcome enabled us to have a much bigger sample size and inclusion of some of the cohorts leading to increased generalizability of the findings. Finally, most of the effect sizes of the two analyses led to very comparable effect estimates. The important limitation of this study is the ascertainment of the medication use at the study visits, and the medication regimen of the participants might have changed during the follow-ups. In addition, we only analysed time to first fall and not a more complex measure such as an incidence rate of falls during a specific time frame or other outcome measures such as falls per hours walked, as this data was not available for all cohorts. Furthermore, although harmonization of cohorts leads to a greater sample size, it also results in some loss of information as more detailed data are reduced to simpler variables in order to achieve harmonization. Finally, assessing many established FRIDs was beyond the scope of this study since their prevalence of use was low. Moreover, we investigated any medication use and did not consider whether the prescriptions were inappropriate as this data was not available in the ADFICE_IT harmonized cohort. 
Future research is warranted regarding the fall risk related to inappropriate prescribing.

\section{Conclusion}

In our propensity score matched approach using data from a harmonized cohort of six European studies, ACE inhibitor and statin users had a lower fall risk when evaluated prospectively. Furthermore, $\beta$-blocker and statin use was associated with a lower fall risk in the past year. Finally, proton pump inhibitor use was associated with a higher fall risk in the past year. In addition, many other medication classes showed no significant associations, indicating that the population-attributable fall risk related to these medication classes on average is low in a relatively healthy older population. In terms of clinical practice, deprescribing of appropriate medication in healthy older persons without side effects is likely not judicious in falls prevention. However, as risk of ADRs such as falls is strongly related to health characteristics such as multimorbidity and frailty, the found results cannot be generalized to frail older adults. Thus, there is a need for studies assessing the risk in a frail population and for studies to identify possible additional subgroups for whom the treatment might be associated with increased risk.

Supplementary Information The online version contains supplementary material available at https://doi.org/10.1007/s40266-021-00876-0.

\section{Declarations}

Funding This work was supported by the Clementine Brigitta Maria Dalderup fund, which is an Amsterdam University fund. The sponsor played no part in the design, execution, analysis and interpretation of data, or writing of the study. The initial B-PROOF study has received funding so far from the Netherlands Organization for Health Research and Development (ZonMw, Grant 6130.0031), The Hague; unrestricted grant from NZO (Dutch Dairy Association), Zoetermeer; Orthica, Almere; Netherlands Consortium Healthy Ageing (NCHA) Leiden/ Rotterdam; Ministry of Economic Affairs, Agriculture and Innovation (project KB-15-004-003), The Hague; Wageningen University, Wageningen; VUmc, Amsterdam; Erasmus Medical Center, Rotterdam. The Longitudinal Aging Study Amsterdam (LASA) is largely supported by a grant from the Netherlands Ministry of Health, Welfare and Sports, Directorate of Long-Term Care. The data collection in 2012-2013 was financially supported by the Netherlands Organization for Scientific Research (NWO) in the framework of the project "New Cohorts of young old in the 21st century" (File Number 480-10-014). The TILDA study is co-funded by the Government of Ireland through the Office of the Minister for Health and Children, by Atlantic Philanthropies and by Irish Life; data were collected under the Statistics Act, 1993, of the Central Statistics Office. The Rotterdam Study is supported by the Erasmus MC University Medical Center and Erasmus University Rotterdam; The Netherlands Organisation for Scientific Research (NWO); The Netherlands Organisation for Health Research and Development (ZonMw); the Research Institute for Diseases in the Elderly (RIDE); The Netherlands Genomics Initiative (NGI); the Ministry of Education,
Culture and Science; the Ministry of Health, Welfare and Sports; the European Commission (DG XII); and the Municipality of Rotterdam. The ActiFE-Indicators for Monitoring COPD [Chronic Obstructive Pulmonary Disease] and Asthma-(Activity and Function in the Elderly in Ulm) study was supported by the European Union (No. 2005121), and the Ministry of Science, Baden-Württemberg, and the German Research Foundation (RO2606/14-1, DE2674/1-1).

Conflicts of interest The authors have no conflicts of interest that are directly relevant to the content of this article.

Ethics approval The studies were performed in line with the principles of the Declaration of Helsinki. All studies were approved by their respective institutional ethics committees [19, 20, 22-24].

Consent to participate All participants provided written informed consent.

Consent for publication Informed consent for publication was obtained as part of initial ethical approval and informed consent procedures.

Availability of data and material Data from the LASA database are available for use for specific research questions provided that an agreement is made up (https://www.lasa-vu.nl/data/availability_data/avail ability_data.htm). Data from TILDA is available through the ISSDA application process (https://www.ucd.ie/issda/data/tilda/). Rotterdam study data can be obtained upon request. Requests should be directed towards the management team of the Rotterdam Study (secretariat. epi@erasmusmc.nl), which has a protocol for approving data requests. Because of restrictions based on privacy regulations and informed consent of the participants, data cannot be made freely available in a public repository. Furthermore, B-PROOF and ActiFE-Ulm study data can be obtained upon request. Requests should be directed towards the principal investigators of the studies, who have a protocol for approving data requests.

Code availability Not applicable.

Author contributions L.S., B.L., N.V., N.S., M.S. and A.A-H contributed to the study conception and design. Material preparation and data collection was performed by all authors. Analysis and/or interpretation of the results were performed by L.S., L.S., M.S., N.V., B.L. and A.A-H. The first draft of the manuscript was written by L.S., M.S., N.V. and A.A-H and all authors commented on previous versions of the manuscript. All authors read and approved the final manuscript.

Open Access This article is licensed under a Creative Commons Attribution-NonCommercial 4.0 International License, which permits any non-commercial use, sharing, adaptation, distribution and reproduction in any medium or format, as long as you give appropriate credit to the original author(s) and the source, provide a link to the Creative Commons licence, and indicate if changes were made. The images or other third party material in this article are included in the article's Creative Commons licence, unless indicated otherwise in a credit line to the material. If material is not included in the article's Creative Commons licence and your intended use is not permitted by statutory regulation or exceeds the permitted use, you will need to obtain permission directly from the copyright holder. To view a copy of this licence, visit http://creativecommons.org/licenses/by-nc/4.0/. 


\section{References}

1. Berry SD, Miller RR. Falls: epidemiology, pathophysiology, and relationship to fracture. Curr Osteoporos Rep. 2008;6:149-54.

2. Centers for Disease Control and Prevention. Home and recreational safety. Important facts about falls. https://www.cdc.gov/ homeandrecreationalsafety/falls/adultfalls.html. Accessed 20 Nov 2020.

3. Peeters G, Bennett M, Donoghue OA, Kennelly S, Kenny RA. Understanding the aetiology of fear of falling from the perspective of a fear-avoidance model-a narrative review. Clin Psychol Rev. 2020;79:101862

4. Merchant RA, Chen MZ, Wong BLL, Ng SE, Shirooka H, Lim JY, et al. Relationship between fear of falling, fear-related activity restriction, frailty, and sarcopenia. J Am Geriatr Soc. 2020;68:2602-8.

5. Bahat Öztürk G, Kılıç C, Bozkurt ME, Karan MA. Prevalence and associates of fear of falling among community-dwelling older adults. J Nutr Health Aging. 2021;25:433-9.

6. Heinrich S, Rapp K, Rissmann U, Becker C, König H-H. Cost of falls in old age: a systematic review. Osteoporos Int. 2010;21:891-902.

7. NICE. NICE guidelines: falls assessment and prevention of falls in older people (CG161). 2013.

8. de Vries M, Seppala LJ, Daams JG, van de Glind EMM, Masud T, van der Velde N, et al. Fall-risk-increasing drugs: a systematic review and meta-analysis: I. Cardiovascular drugs. J Am Med Dir Assoc. 2018;19:371 e1-e9.

9. Seppala LJ, Wermelink A, de Vries M, Ploegmakers KJ, van de Glind EMM, Daams JG, et al. Fall-risk-increasing drugs: a systematic review and meta-analysis: II. Psychotropics. J Am Med Dir Assoc. 2018;19:371.e11-e17.

10. Seppala LJ, van de Glind EMM, Daams JG, Ploegmakers KJ, de Vries M, Wermelink A, et al. Fall-risk-increasing drugs: a systematic review and meta-analysis: III. Others. J Am Med Dir Assoc. 2018;19(372):e1-8

11. Mangin D, Bahat G, Golomb BA, Mallery LH, Moorhouse P, Onder $\mathrm{G}$, et al. International Group for Reducing Inappropriate Medication Use \& Polypharmacy (IGRIMUP): position statement and 10 recommendations for action. Drugs Aging. 2018;35:575-87.

12. O'Mahony D, O'Sullivan D, Byrne S, O'Connor MN, Ryan C, Gallagher P. STOPP/START criteria for potentially inappropriate prescribing in older people: version 2. Age Ageing. $2015 ; 44: 213-8$.

13. Bahat G, Ilhan B, Erdogan T, Halil M, Savas S, Ulger Z, et al. Turkish inappropriate medication use in the elderly (TIME) criteria to improve prescribing in older adults: TIME-to-STOP/TIMEto-START. Eur Geriatr Med. 2020

14. American Geriatrics Society. Updated beers criteria for potentially inappropriate medication use in older adults. J Am Geriatr Soc. 2015;2015(63):2227-46.

15. Seppala LJ, van der Velde N, Masud T, Blain H, Petrovic M, van der Cammen TJ, et al. EuGMS Task and Finish group on Fall-Risk-Increasing Drugs (FRIDs): position on knowledge dissemination, management, and future research. Drugs Aging. 2019;36:299-307.

16. Summary of the Updated American Geriatrics Society/British Geriatrics Society clinical practice guideline for prevention of falls in older persons. J Am Geriatr Soc. 2011;59:148-57.

17. Glynn RJ, Schneeweiss S, Stürmer T. Indications for propensity scores and review of their use in pharmacoepidemiology. Basic Clin Pharmacol Toxicol. 2006;98:253-9.

18. Onderzoekslijn Gepersonaliseerde val- \& fractuurpreventie. ADFICE_IT. 2020. https://www.amc.nl/web/specialismen/geria trie-ouderengeneeskunde-1/onderzoekslijn-gepersonaliseerde-valfractuurpreventie/valpreventie-1/adfice_it.htm. Accessed 26 Nov 2020.

19. Ikram MA, Brusselle GGO, Murad SD, van Duijn CM, Franco $\mathrm{OH}$, Goedegebure A, et al. The Rotterdam Study: 2018 update on objectives, design and main results. Eur J Epidemiol. 2017;32:807-50.

20. van Wijngaarden JP, Dhonukshe-Rutten RAM, van Schoor NM, van der Velde N, Swart KMA, Enneman AW, et al. Rationale and design of the B-PROOF study, a randomized controlled trial on the effect of supplemental intake of vitamin B12and folic acid on fracture incidence. BMC Geriatr. 2011;11:80.

21. Swart KM, Ham AC, van Wijngaarden JP, Enneman AW, van Dijk $\mathrm{SC}$, Sohl E, et al. A randomized controlled trial to examine the effect of 2-year vitamin B12 and folic acid supplementation on physical performance, strength, and falling: additional findings from the B-PROOF study. Calcif Tissue Int. 2016;98:18-27.

22. Hoogendijk EO, Deeg DJH, de Breij S, Klokgieters SS, Kok AAL, Stringa N, et al. The Longitudinal Aging Study Amsterdam: cohort update 2019 and additional data collections. Eur J Epidemiol. 2020;35:61-74.

23. Denkinger MD, Franke S, Rapp K, Weinmayr G, Duran-Tauleria E, Nikolaus T, et al. Accelerometer-based physical activity in a large observational cohort-study protocol and design of the activity and function of the elderly in Ulm (ActiFE Ulm) study. BMC Geriatr. 2010;10:50.

24. Donoghue OA, McGarrigle CA, Foley M, Fagan A, Meaney J, Kenny RA. Cohort profile update: the Irish Longitudinal Study on Ageing (TILDA). Int J Epidemiol. 2018;47:1398-1.

25. Ham AC, Ziere G, Broer L, Swart KM, Enneman AW, van Dijk SC, et al. CYP2C9 Genotypes modify benzodiazepine-related fall risk: original results from three studies with meta-analysis. J Am Med Dir Assoc. 2017;18:88.e1-e15.

26. WHO Collaborating Centre for Drug Statistics Methodology. ATC/DDD Index 2020. 2020. https://www.whocc.no/atc_ddd_ index/. Accessed 20 Nov 2020.

27. Aarts N, Noordam R, Hofman A, Tiemeier H, Stricker BH, Visser LE. Self-reported indications for antidepressant use in a population-based cohort of middle-aged and elderly. Int J Clin Pharm. 2016;38:1311-7.

28. Ham AC, Enneman AW, van Dijk SC, Oliai Araghi S, Swart KM, Sohl E, et al. Associations between medication use and homocysteine levels in an older population, and potential mediation by vitamin B12 and folate: data from the B-PROOF Study. Drugs Aging. 2014;31:611-21.

29. Huisman M, Poppelaars J, van der Horst M, Beekman AT, Brug J, van Tilburg TG, et al. Cohort profile: the Longitudinal Aging Study Amsterdam. Int J Epidemiol. 2011;40:868-76.

30. Peklar J, Kos M, O’Dwyer M, McCarron M, McCallion P, Kenny RA, et al. Medication and supplement use in older people with and without intellectual disability: an observational, cross-sectional study. PLoS One. 2017;12:e0184390.

31. McMurry TL, Hu Y, Blackstone EH, Kozower BD. Propensity scores: Methods, considerations, and applications. J Thorac Cardiovasc Surg. 2015;150:14-9.

32. White IR, Royston P, Wood AM. Multiple imputation using chained equations: Issues and guidance for practice. Stat Med. 2011;30:377-99.

33. Federatie Medisch Specialisten. Richtlijn Preventie van valincidenten bij ouderen. (Dutch guideline prevention of falls in older people). 2017.

34. The R Project for Statistical Computing. https://www.r-project. org/. Accessed 20 Nov 2020.

35. Seppala LJ, Petrovic M, Ryg J, Bahat G, Topinkova E, Szczerbińska K, et al. STOPPFall (Screening Tool of Older Persons Prescriptions in older adults with high fall risk): a Delphi 
study by the EuGMS Task and Finish Group on Fall-Risk-Increasing Drugs. Age Ageing. 2020.

36. Poly TN, Islam MM, Yang HC, Wu CC, Li YJ. Proton pump inhibitors and risk of hip fracture: a meta-analysis of observational studies. Osteoporos Int. 2019;30:103-14.

37. Shah BR, Laupacis A, Hux JE, Austin PC. Propensity score methods gave similar results to traditional regression modeling in observational studies: a systematic review. J Clin Epidemiol. 2005;58:550-9.
38. Jansen S, Bhangu J, de Rooij S, Daams J, Kenny RA, van der Velde N. The association of cardiovascular disorders and falls: a systematic review. J Am Med Dir Assoc. 2016;17:193-9.

39. Mol A, Bui Hoang PTS, Sharmin S, Reijnierse EM, van Wezel RJA, Meskers CGM, et al. Orthostatic hypotension and falls in older adults: a systematic review and meta-analysis. J Am Med Dir Assoc. 2019;20:589-97.e5.

40. Alomar MJ. Factors affecting the development of adverse drug reactions (review article). Saudi Pharmaceut J. 2014;22:83-94.

\section{Authors and Affiliations}

\section{J. Seppala ${ }^{1}$ B. van de Loo ${ }^{1,3} \cdot$ M. Schut ${ }^{2} \cdot$ N. M. van Schoor ${ }^{3}$ B. H. Stricker ${ }^{4}$ R. A. Kenny ${ }^{5}$ F. Moriarty ${ }^{5,6}$. L. C. P. G. M. de Groot ${ }^{7} \cdot$ M. Denkinger ${ }^{8} \cdot$ D. Rothenbacher ${ }^{9} \cdot$ Nathalie van der Velde $^{1}$ (1) $\cdot$ A. Abu-Hanna ${ }^{2}$}

1 Section of Geriatric Medicine, Department of Internal Medicine, Amsterdam Public Health Research Institute, Amsterdam UMC, University of Amsterdam, Meibergdreef 9, 1105AZ Amsterdam, The Netherlands

2 Department of Medical Informatics, Amsterdam Public Health Research Institute, Amsterdam UMC, University of Amsterdam, Meibergdreef 9, 1105AZ Amsterdam, The Netherlands

3 Department of Epidemiology and Data Science, Amsterdam UMC, Vrije Universiteit Amsterdam, Amsterdam Public Health Research Institute, Amsterdam, The Netherlands

4 Department of Epidemiology, Erasmus University Medical Center, Rotterdam, The Netherlands
5 Department of Medical Gerontology, TILDA, Trinity College, Dublin, Ireland

6 HRB Centre for Primary Care Research, Royal College of Surgeons in Ireland, 123 St Stephen's Green, Dublin D02 YN77, Ireland

7 Division of Human Nutrition and Health, Wageningen University, Wageningen, The Netherlands

8 Geriatric Research Unit, Agaplesion Bethesda Clinic, Geriatric Centre Ulm, Ulm University, Ulm, Germany

9 Institute of Epidemiology and Medical Biometry, Ulm University, Ulm, Germany 